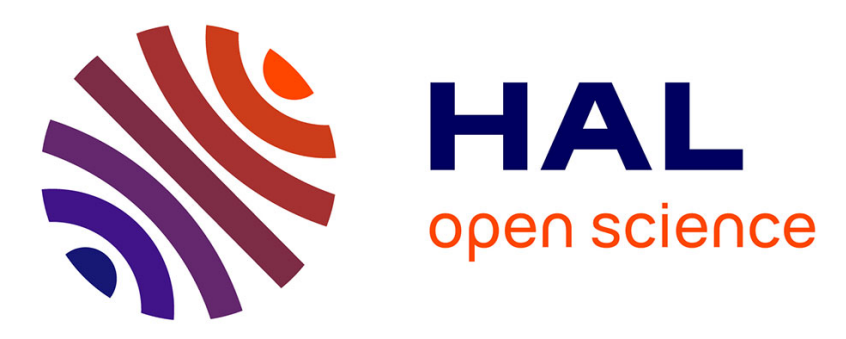

\title{
On the role of stochastic geometry in sixth generation wireless networks
}

\author{
Yassine Hmamouche, Mustapha Benjillali, Samir Saoudi
}

\section{To cite this version:}

Yassine Hmamouche, Mustapha Benjillali, Samir Saoudi. On the role of stochastic geometry in sixth generation wireless networks. ISIVC 2020: 10th International Symposium on Signal, Image, Video and Communications, Apr 2021, Saint-Etienne, France. 10.1109/ISIVC49222.2021.9487542 . hal02897827

\section{HAL Id: hal-02897827 \\ https://imt-atlantique.hal.science/hal-02897827}

Submitted on 26 Aug 2020

HAL is a multi-disciplinary open access archive for the deposit and dissemination of scientific research documents, whether they are published or not. The documents may come from teaching and research institutions in France or abroad, or from public or private research centers.
L'archive ouverte pluridisciplinaire HAL, est destinée au dépôt et à la diffusion de documents scientifiques de niveau recherche, publiés ou non, émanant des établissements d'enseignement et de recherche français ou étrangers, des laboratoires publics ou privés. 


\title{
On the Role of Stochastic Geometry in Sixth Generation Wireless Networks
}

\author{
Yassine Hmamouche ${ }^{1,2}$, Mustapha Benjillali ${ }^{2}$, and Samir Saoudi ${ }^{1}$ \\ ${ }^{1}$ IMT Atlantique, Lab-STICC, UBL, 29238 Brest, France \\ Emails: \{yassine.hmamouche; samir.saoudi\}@imt-atlantique.fr \\ ${ }^{2}$ Communication Systems Department, INPT, Rabat, Morocco \\ Email: benjillali@ieee.org
}

\begin{abstract}
Over the past four decades, the mobile communications industry has evolved from the first generation (1G) to the 5G premises, while the research community is currently discussing the broad outlines of the 6G system foreseen from 2030 onwards. In fact, even if $5 \mathrm{G}$ is extensively advocated as the expected ecosystem to fully meet the requirements of internet of things (IoT) applications, e.g., autonomous robots and vehicles, extended reality (XR) platforms, and brain-computer interface (BCI), it is only by the advent of 6G that such a bold achievement can be truly met. One key milestone towards the $6 \mathrm{G}$ vision is the development of a multi-objective analytical tool enabling the joint optimization of communication, control, localization, sensing, energy, and other parameters and resources. In this paper, we discuss how stochastic geometry (SG) can serve as a potential candidate for such aim. We also outline some fundamental limitations of SG, as well as some avenues to address them, and develop new seminal results ready-to-use in the analysis and modeling of future 6G networks.
\end{abstract}

\section{INTRODUCTION}

To keep pace with societal technology shifts, and the neverending quest to send more data, more quickly, and more efficiently over the air, a new generation of cellular standards is approximately required to emerge every decade [1].

As illustrated in Tab. I, 1G wireless cellular technology was actually introduced in the 1980s such that a voice signal is analog-modulated at a higher frequency, typically 150 $\mathrm{MHz}$ and more. The second generation (2G) of wireless communications appeared around 1991, where information signals are digitally transmitted, making it possible to send, for the first time, text messages over a cellular network. In the year 2000, the third generation (3G) of mobile networks was commercially rolled out, offering faster data transfer speeds of more than $384 \mathrm{kbps}$ and laying the groundwork for wireless internet access. In 2012, the first 4th generation networks were deployed, offering significantly higher data rates than $3 \mathrm{G}$, with the possibility of transferring voice calls over a fully IP-based core network. The benefits of $4 \mathrm{G}$, combined with the rapid advances in smartphones and tablets technology, have allowed end users to install various software applications from a centralized store, which broadens the scope of $4 \mathrm{G}$ use cases and paves the way to a fully digitalized society.

This work is funded by a research grant from PRACOM and the Regional Council of Brittany, France.
While previous generations of cellular networks (from $1 \mathrm{G}$ to $4 \mathrm{G}$ ) were originally designed to deliver voice and rate-centric mobile internet access services, the fifth generation (5G) is engineered in part to provide enhanced mobile broadband (eMBB) capabilities enabling seamless user experience for streaming video, truly immersive virtual reality (VR) and augmented reality (AR) applications, and more. In addition, $5 \mathrm{G}$ is typically aimed to support the requirements of low latency and high reliability that are necessary to connect billions of peoples and machines in the internet of things (IoT) fashion [2]. IoT applications are typically divided into two categories, the first one is related to ultra-reliable low-latency communications (URLLC) to support critical applications that require robust and uninterrupted data transfer, e.g., factory automation, smart grids, autonomous vehicles, robot-enabled remote surgery. The second category is massive machine-type communications (mMTC) enabling massive connectivity of intelligent devices, e.g., smart sensors, cities.

Typically, several coordinated efforts to standardize $5 \mathrm{G}$ have been conducted at the first milestone of the new $5 \mathrm{G}$ radio (5G NR) and later versions of 3rd generation partnership project (3GPP). The first 5G standard is Release 15 (R15) frozen in June 2018, which is particularly driven towards improving the requirements for eMBB, while for $\mathrm{mMTC}$ and URLLC, it re-uses the narrowband IoT (NB-IoT) based on long term evolution (LTE) and enhanced MTC (eMTC) in 3GPP R14, which only offers similar reliability and latency capabilities for URLLC as the eMBB [3]. Hence, despite $5 \mathrm{G}$ is extensively advocated as the expected ecosystem to perfectly meet the IoT requirements for mMTC and URLLC, it is actually unlikely to be fully achieved in the very near future. Instead, several experts agree that a ten-year horizon on the eve of $5 \mathrm{G}$ deployment is required to truly envision and build a 6th generation (6G) system that can meet the full requirements of IoT applications (massive connectivity, the emergence of robots and unmanned vehicles, brain-computer interface (BCI), extended reality (XR) platforms), and their associated technology trends (edge computing, artificial intelligence (AI), large intelligent surfaces (LIS), the use of tremendously higher frequency bands) [3]-[5].

Accordingly, a vital component towards the 2030s vision of the $6 \mathrm{G}$ system, is the development of an analytical tool 


\begin{tabular}{|l|l|l|l|l|l|l|}
\hline Technology generation & $\mathbf{1 G}$ & $\mathbf{2 G}$ & $\mathbf{3 G}$ & $\mathbf{4 G}$ & $\mathbf{5 G}$ & $\mathbf{6 G}$ \\
\hline Operational year & 1984 & 1991 & 2002 & 2012 & 2020 & 2030 \\
\hline Peak data rate & $2.4 \mathrm{kbps}$ & $64 \mathrm{kbps}$ & $2 \mathrm{Mbps}$ & $100 \mathrm{Mbps}$ & $10 \mathrm{Gbps}$ & $1 \mathrm{Tbps}$ \\
\hline Wireless access & Analog & Digital & Digital & Digital & Digital & Digital \\
\hline Core network & Circuit-based & Circuit-based & $\begin{array}{l}\text { Circuit and } \\
\text { packet-based }\end{array}$ & Fully IP-based & Fully IP-based & Fully IP-based \\
\hline Key services & $\begin{array}{l}\text { Mobile } \\
\text { telephony }\end{array}$ & $\begin{array}{l}\text { Digital voice, } \\
\text { short messages }\end{array}$ & Internet & Internet of applications & Internet of things & $\begin{array}{l}\text { A fully intelligent } \\
\text { and connected world }\end{array}$ \\
\hline
\end{tabular}

TABLE I

Mobile COMmUnicATion: From 1G To 6G

allowing to prevent onerous and time-consuming computer simulations, and also multi-objective analysis of data rate, latency, and reliability, which ultimately drives the joint optimization of communication, control, localization, sensing, energy, and many others.

This paper is a projected vision, in which we aim to i) outline the core elements of the $6 \mathrm{G}$ vision, including an overview of the international research and development (R\&D) initiatives, the potential use cases, and the key enabling technologies, ii) discuss why SG can serve as a powerful mathematical tool for performance evaluation of $6 \mathrm{G}$ wireless networks, iii) review some key shortcomings of using such analytical tool, and iv) suggest new research avenues that may overcome the limitations of SG and help to develop new seminal results ready-to-use in performance analysis of future $6 \mathrm{G}$ networks.

\section{II. $6 \mathrm{G}$ VISIONS}

In this section, we will outline the core elements of the 2030 s vision of $6 \mathrm{G}$ system.

\section{A. International $6 G R \& D$ Initiatives}

Several international organizations and economic actors have initiated the study of $6 \mathrm{G}$. In Europe, the international telecommunication union (ITU) has created a new discussion group to guide the global information and communications technology (ICT) community in studying network capacity for the year 2030 and beyond [6]. The European technology platform for communications networks and services (https://www.networld2020.eu/) has launched in 2017 a research project to think about next big steps of communication in beyond $5 \mathrm{G}$ (B5G) and the types of new services that will impact people's lives by the end of this decade. In April 2018, the academy of Finland announced the official start of the first $6 \mathrm{G}$ R\&D Flagship project with a budget of $€ 251$ million, "6Genesis", to conceptualize 6G through a joint effort of the university of Oulu and Nokia [7]. A first result is the publication of the world's first white paper on $6 \mathrm{G}$, describing the main drivers, requirements, and research challenges [8]. More recently, the UK government has invested $£ 15$ million to develop research in quantum technologies for $6 \mathrm{G}$ networks from smartphones to quantum computers (https://www.gov.uk). In Asia, south Korean groups such as
LG and Samsung have announced plans for 6G R\&D. In China, where there is already the largest deployed 5G network, the government has made up a working group of government departments as well as academic and scientific experts to distill recommendations in $6 \mathrm{G}$ R\&D (www.china.org.cn). Farther afield, some universities in the USA have initiated research on the merits of exploiting terahertz waves for $6 \mathrm{G}$ wireless networks (wireless.engineering.nyu.edu). In general, as with $5 \mathrm{G}, 6 \mathrm{G}$ research will necessarily be based on open innovation and international cooperation. As such, international partnerships are expected to emerge, for instance, the memorandum signed in 2019 between the South Korean government and the University of Oulo to cooperate in the development of $6 \mathrm{G}$. Also, Sony, NTT, and Intel have announced their intention to form a strategic partnership for the development of advanced semiconductor technology for $6 \mathrm{G}$ networks.

\section{B. Potential Use Cases}

Massive connectivity: An important requirement of a $6 \mathrm{G}$ system is its ability to handle massive volumes of data and high-speed connectivity per device. According to ITU forecasts [6], the number of global mobile subscriptions will increase by more than 6 billion over the period 2020-2030, while mobile traffic is expected to grow by an annual rate of about $54 \%$ over the same period (4,394 Exabyte in 2030 compared to 57 Exabyte in 2020). These unprecedented expansions are indeed driven by many factors, including i) the improved performance of mobile internet (eMBB) compared to fixed internet via cable and fiber, ii) the shift in traffic consumption patterns with a strong prevalence of video streaming, iii) increasing demand for mobile services in the cloud, as users increasingly adopt services that need to be pervasive, iv) the increased availability of high-resolution video and audio content, v) the expected development of devices with enhanced capabilities such as wearable displays and smart body implants that are likely to doom the age of smartphones, vi) last but not least, the evolution of worldwide demography, urban migration and the increasing trend towards urbanization.

The emergence of robots and unmanned vehicles: 6G networks are envisioned to be heavily driven by emerging artificial intelligence (AI) technologies such as deep learning, computer vision, and natural language processing (NLP), which promises the emergence of autonomous and intelligent 
robots in land, sea, and sky, most notably in applications entailing repetitive tasks, from transportation and logistics to medical surgery. Typically, the traditionnal terrestrial heterogeneous network (HetNet) will be rolled out to the sky by integrating unmanned areal vehicles (UAVs), high altitude platforms (HAPs), and even sophisticated satellites, which will enable a super-connectivity paradigm by supporting coverage in isolated areas and zones with heavy traffic [9], [10]. Autonomous robots are also being extensively used in the process of production automation to support a new generation of connected, robotic, and intelligent factories, referred to as the Industry 4.0 concept. Ensuring such ecosystem requires high eMBB performance for voice and HD image/map processing, in addition to a perfect reliability (even in extreme weather conditions) with regard to the algorithms and sensors fitted on robots for complete remote control and satellite positioning.

Brain-computer alchemy: An exciting vision of future ultra-high-speed $6 \mathrm{G}$ data networks is their ability to integrate advanced brain-computer interface (BCI) technologies, which will substantially improve human life and build an intelligent environment [5]. "When you look at $1 G$ being for voice, for your ears. And $3 G$ through $5 G$ are data for your eyes, for a vision service. perhaps $6 G$ will go beyond the head mounted displays to introduce direct neural interface,"Qualcomm chief technology officer Matt Grob commented at Quartzs The Next Billion conference held in San Francisco on October 2016.

A BCI is actually a set of low-powered brain sensors used to capture signals from the brain, process them, and translate them into commands that are transmitted to an external machine, allowing the brain to merge its power to that of the machine. Generally, recent applications of BCI are typically organized into three broad areas, which are mental typewriting, rehabilitation engineering, and augmented cognition. A more advanced version of the BCI is the Bi-directional BCI (BBCI), which enables simultaneous harvesting of commands from the brain and stimulus of the brain from the external environment endowing it with capabilities not accessible to ordinary humans, e.g., extending vision to near-IR range or U.V. range, and improving auditory capabilities to very low decibel or high frequency sounds. However, major challenges in BCI research are generally related to the inherent attributes of brain signals, namely, very low voltage (magnitude of $\mathrm{mV}$ ), high level of variability, as well as the non-stationarity, which requires the development of very powerful sensors and sturdy classification algorithms by 2030s.

The extended reality (XR) platforms: One of the key experiences that will disrupt our daily tasks in the vision of 2030s is the $\mathrm{XR}$, intended to actually combine the augmented reality (AR), virtual reality (VR), and mixed reality (MR) technologies [11]. AR consists of overlaying digital information onto the real world, while VR consists in completely substituting one reality by another, entirely digital (sounds and images). As for MR, it entails enriching the real world with rich interactive digital elements with real-time environment. 3GPP R16 has launched a feasibility study of XR in the context of 5G [12].
The scope of the document is to introduce the core technology enablers, key use cases, performance metrics for XR services, as well as potential needs for standardization in 3GPP. In general, the real-time interactive nature of $\mathrm{XR}$ applications requires combined excellent performance in: eMBB (at least $100 \mathrm{MBps}$ ) to receive large amounts of information in real time (HD maps, $360^{\circ}$ video streaming), URLLC (with less than $1 \mathrm{~ms}$ latency) for an excellent XR user experience when relocating, powerful edge computing to ensure intelligent data processing as close as possible to end devices, and excellent energy efficiency at end devices. However, the 5G NR is typically focused on improving downlink and uplink eMBB services where disruptive enhancement of the mobile latency required for $\mathrm{XR}$ applications is a real battleground that may only be fully won in the $6 \mathrm{G}$ era.

\section{Key Enabling Technologies}

Towards ubiquitous edge intelligence: The steady growth in traffic volumes required by 2030s services [6], along with the enormous processing capability required to analyze these massive amounts of data, is likely to create significant congestion at backhaul links and then latency impairment. A milestone in addressing such limitation is by combining the strengths of two major technologies, namely i) the Fog radio access network (F-RAN) architecture [13], in which a handful of the cloud is deployed in the vicinity of end users, which can be done by equipping user terminals or third entities (e.g. parks, shopping centers) with computing and storage capacity. ii) AI with its powerful learning, analysis, and optimization capabilities, which enables 6G network architectures to learn and cope with the most complex situations by reducing human intervention as much as possible. Generally, we note in particular the use of machine learning (ML) and deep learning (DL) as AI popular techniques for the analysis of wireless networks.

Towards smart propagation environments: Signal propagation in the wireless environment of $6 \mathrm{G}$ networks will also be endowed with intelligence where the channel model is no longer treated as a random probabilistic process, but as a software-defined metric enabled by large intelligent surfaces (LIS) [14]. Indeed, LIS enables a radically new wireless communication paradigm, in which certain software-controlled metal reflectors, made of low-cost passive elements, namely metasurfaces, are judiciously placed in ambient objects and controlled by the power of AI in order to minimize signal fading during reflections. Metasurfaces are in fact equipped with atomic elements exhibiting a periodic texture, whose geometric characteristics can be controlled to change the direction of the incident electromagnetic waves. One of the major challenges facing massive adoption of LIS is actually how to quantify the benefits of using LIS on the performance of large-scale wireless networks, e.g., coverage probability, ergodic rate, and energy efficiency.

Higher frequency bands beyond sub-6 GHz: With the acute spectrum scarcity in commercial wireless networks currently running in frequency bands below $6 \mathrm{GHz}$, researchers are exploring new avenues in higher bands to ensure sufficient 
bandwidth and meet the increased data throughput requirements for eMBB services. In particular, the subsequent bands are expected to play an important role in achieving the 1 Tbps target of the 2030s vision. Namely, i) the popular mmWave and TeraHertz communications with respective wavelengths of 1 to $10 \mathrm{~mm}$ and $100 \mathrm{~m}$ to $1 \mathrm{~mm}$, and frequency bands of 30 to 300 $\mathrm{GHz}$ and $300 \mathrm{GHz}$ to $3 \mathrm{THz}$, iii) visible light communication (VLC) using light-emitting diodes in the unlicensed visible light spectrum between 400 to $800 \mathrm{THz}$ and wavelengths of 375 to $780 \mathrm{~nm}$, and iv) free-space optical (FSO) communications that use signals with wavelength in 785-1550 $\mathrm{nm}$, it is commonly used as a laser beam for high-speed data transmissions in a point-to-point free space environment, where it can be used as a promising backhaul solution to avoid impractical deployments of wired connections.

\section{Stochastic GeOMETRY AS A NECESSARY ANALYTICAL TOOL FOR PERFORMANCE EVALUATION OF 6G WiRELESS NETWORKS}

From previous discussions, it is obvious to mention that 6G networks are going to be highly heterogeneous, multilayered, with embedded intelligence at both the core and edge of the network, where AI is expected to play a crucial role in link and system level decisions. Also, performance metrics will be carefully tailored to ensure joint evaluation of multiple metrics, typically the joint optimization of data rate, latency, and reliability, which ultimately drives the joint optimization of communication, control, localization, sensing, energy, and many others. Therefore, system-level performance evaluation of $6 \mathrm{G}$ networks will be valuable to formulate relevant insights into tradeoffs that govern such a system. This section discusses i) why SG can serve as a powerful mathematical tool for system-level performance analysis of future $6 \mathrm{G}$ networks, ii) as well as some of its fundamental limitations.

\section{A. Inherent SG Capabilities for Analysis and Modeling of $6 G$ Wireless Networks}

SG to capture the spatial arrangement of nodes in 6G wireless networks: $6 \mathrm{G}$ networks are widely intended to support a myriad of user equipment (smart phones, body implants, displays, XR devices, aerial users) with very diverse technical characteristics (transmission power, communication mode, agility and more). Similarly, base stations (BSs) can also range from the conventional macro, micro, and pico terrestrial BSs, to emerging configurations including aerial BSs (UAV, HAP, and satellites), along with spatial diversity technologies such as repeaters, LIS, and device-to-device (D2D) communications. In such a heterogeneous and complex landscape, the signal-to-interference-and-noise ratio (SINR), considered as a first-order predictor of users quality of service (QoS), will always be tightly correlated with the geometric distribution of the transmitter-receiver distance. Accordingly, SG as a field of applied probability can provide tractable mathematical models and appropriate statistical methods to study and analyze such typical random distances. For instance, multi-tier transmitters and receivers are conceived as scattered randomly over an enormous number of possibilities, and their locations is assessed statistically in order to evaluate spatial averages of network performance connectivity.

SG in the era of machine learning: With the unprecedented availability of data, requiring software-controlled and optimized operations, besides the recent advances in F-RAN and intelligent radio environments through the use of metasurfaces, AI and more typically ML are poised to play a major role in managing these complex scenarios where optimizations have to be performed under an almost unlimited number of degrees of freedom and system constraints. Interestingly, SG can offer a common ground and a potential for crossfertilization with ML, so that the merits of the two approaches will be jointly exploited to address similar problems. Indeed, SG models will be embedded as a class of hypothesis in the learning process of ML. An illustrative case is the class of problems known as the subset selection problems, where an optimal subset must be selected from a baseline set. In such a context, SG probabilistic models constructed by finite determinantal point process (DPP) are used to feed ML data-driven supervised learning frameworks. The arts in [15], [16] represent initial contributions in this direction of crossfertilization between SG and ML.

SG for the evaluation of higher frequency bands: SG has demonstrated its ability and relevance to analyze system setups involving high frequency bands [17]-[20]. This requires in particular some key adjustments to the modeling choices considered in the SG frameworks. For instance, i) nodes need to be equipped with directional antennas where the antenna gain is maximized when the steering angle is inside a given main lobe width. ii) the vulnerability of higher frequency bands to blockages is captured by considering LOS and NLOS transmissions in addition eventually to an outage state, in which the path loss is approximately infinite. iii) The primacy of the thermal noise w.r.t. the interference in higher frequency bands, is captured by assuming the noise-limited regime. iv) Due to the increased propagation losses in higher frequency bands given the severe sensitivity to rain and resonant absorption in water molecules, the path loss function needs to be generally revisited by a frequency-dependent molecular absorption effect with an emphasis on the LOS link as

$$
\ell(r)=\left(\frac{c}{4 \pi f}\right)^{2} r^{-\alpha_{\mathrm{LOS}}} \mathrm{e}^{-k(f) r}
$$

where $k(f)$ is the medium absorption coefficient at frequency $f, \alpha_{\mathrm{LOS}}$ is the path loss exponent in the LOS region, and $c$ is the speed of light. v) the transmitted signal can also be constrained by fluctuations in temperature and humidity gradients over time, also known as scintillation or turbulenceinduced fading, a universal model to capture such turbulence conditions is to consider doubly stochastic fading models [20].

SG and brain-computer interaction: In the 6G system vision, BCI will indeed enable to merge the enormous internal and external information streams managed by the human nervous system with the conventional wireless network. The BCI can basically process the information generated by neurons in 
their electrical form, while an advanced paradigm is to manage neurons information in their molecular form. In fact, motivated by advances in nanotechnology and molecular communication, biocompatible nanoscale sensors can be implanted into the human body and be able to interact directly with the body's cells, organs, and tissues. That is, molecules are the basic carriers of information, and their position is not deterministic but managed by a random Brownian motion via inherent thermal energy. In this context, a probabilistic analytical tool like SG may be considered as very necessary to estimate the likelihood of finding swarms of particular molecules in a given location of the body and then evaluate the performance of such a miniaturized communication system [21]. That is, such analysis will be valuable to compensate for the laborious and expensive nature of laboratory experimentation of molecular communications.

SG to evaluate the effect of uncontrolled phenomenas: Due to the expected role of emerging technologies such as AI and F-RAN, as well as LIS allowing to even control the propagation environment via AI-based operations rather than a random environment represented by random variables (multipath fading, shadowing), it is envisioned that the $6 \mathrm{G}$ system will enable a highly intelligent and efficient control of massive amounts of information and devices, enabling perfectly controlled and supervised operations, and then a selfoptimization of the $6 \mathrm{G}$ system's utility functions. However, despite all this pervasive intelligence, it is almost unfeasible or even impossible to control all or at least "perfectly all" the components of the $6 \mathrm{G}$ system. For instance, building sway generated by winds and thermal expansion of materials or beams misalignment in higher-frequency communications [22]. Hence, the ubiquitous need to model such uncontrolled network aspects with random processes via SG theory.

\section{B. Some Stochastic Geometry Limitations}

The trade-off between accuracy and tractability: SG, as a powerful model-based approach conceived to improve the tractability and accuracy of traditional probabilistic models (e.g. channel, interference, scheduling), is generally governed by a trade-off between tractability and accuracy. However, with the unprecedented availability of data, the growing heterogeneity, and complexity of future communication systems, SG tractable models will be simply so superficial to capture such realistic scenarios (i.e., unlimited degrees of freedom and system constraints), while accurate models will be very challenging to derive and their resulting algorithms are too complex to deploy. New insights are therefore needed to address this embarrassing trade-off between tractability and accuracy that affects SG models.

The question of the Crofton cell: Point process (PP) theory has a prominent role in SG as points are in general the building blocks of advanced geometric shapes. That is, we note in particular the relevance of the Poisson point process (PPP) that enables to derive tractable expressions of network performance, while other beyond-PPP enable increased modeling accuracy with reduced mathematical flexibility. Furthermore, we often consider in SG the typical point concept to evaluate the performance of PPP wireless networks. Formally, it is a point that has been chosen by a selection procedure in which each point in the process has the same chance of being selected. In particular, one is interested in evaluating the contribution of the typical cell to the coverage of a typical user. However, due to the complexity to obtain such a rigorous result, most of the literature works assimilate such coverage contribution to that of the cell where the typical user exists, known as the 0-cell or the Crofton cell, Which gives optimistic results of the performance. Typical efforts to approximate the downlink coverage of the typical cell are recently proposed in [23].

Average-based vs fine-grained analysis: SG provides powerful analytical tools for evaluating wireless networks with randomly placed nodes (transmitters and/or receivers), where key performance metrics (coverage probability, ergodic rate) are derived by spatial averaging over infinite realizations of the PP capturing nodes location. While this average-based approach is certainly important, yielding a macro-scale view of performance per average user and per unit area, it does not however provide information on the performance concentration per single links or per regions. Interestingly, the concept of meta-distribution is proposed in [24] allowing to quantify the variability of network performance and obtain a finegrained information about it. However, the meta-distribution is restricted to the interference-limited regime while $6 \mathrm{G}$ networks are expected to operate in noise-limited regime given the use of higher frequency bands. Also, reshaping the meta-distribution is generally so complicated and reduces to an instance of the Hausdorff moment problem, i.e., derive the inverse of the $k$ moment.

\section{Towards New Directions Based on Stochastic GEOMETRY}

Previous discussions highlighted the inherent merits of SG to play an essential role in future $6 \mathrm{G}$ networks. Nevertheless, $\mathrm{SG}$ is also suffering from some analytical challenges that may inhibit its usefulness. In this section, two prominent theories are discussed to upgrade $\mathrm{SG}$, and pave the way for new SG seminal results ready-to-use in the analysis and modeling of future wireless networks.

\section{A. Random Fractals for Self-Similarity attributes and Multi- Objective Optimization}

As 6G networks are projected to increase in complexity spanning all levels of communication, a thorough knowledge of the geometrical properties driving the components of this global communication process, which are ranged by their design attributes (e.g., volume, motion) from the atomic scale (BCI, molecular communications) to the aerial scale (UAVs, HAPs, satellites), is necessary to fully grasp the system-level performance of $6 \mathrm{G}$ networks. That is, mathematicians have developed powerful concepts to capture and analyze these complexity scenarios. In particular, random fractals, initially introduced by Mandelbrot in [25], can be used to describe 
similar characteristics (self-similarity) at various scales, and next enable to capture extremely natural and sophisticated setups. Interestingly, fractals are not merely confined to study the essence of geometrical processes, but can also inspire applications in temporal processes (user traffic, mobility setups), and even in learning processes within ML recognition algorithms. Interestingly, fractals can also be used to guide the development of multi-objective optimization algorithms with improved convergence performance for 6G system [26].

\section{B. Grothendieck Toposes to Create Mathematical Bridges to Stochastic Geometry}

Grothendieck topos is a powerful mathematical concept introduced by Alexander Grothendieck during his seminar on algebraic geometry in the early 1960s. The main idea is to create a vast mathematical thing that can create a common field of interaction between the most distant mathematical fields. According to Olivia Caramello [27], Grothendieck toposes can be considered as unifying spaces where properties, ideas, and results can be transferred between distinct mathematical theories. In this case, between SG and other mathematical strands, so that long-standing problems formulated in SG can be solved using methods from a different field where we have reached a good degree of expertise and proficiency.

\section{CONCLusion}

This paper represents a contribution to the burgeoning discussions around the future $6 \mathrm{G}$ wireless networks, expected around 2030s to enable a super-connectivity paradigm with pervasive embedded intelligence.

We have then summarized the key issues of 6G technology including the key $6 \mathrm{G}$ international R\&D initiatives, the potential use cases, and the key enabling technologies such as AI, F-RAN, smart propagation environments via metasurfaces, and the use of higher frequency bands. Next, we have discussed the merits and limitations of $\mathrm{SG}$ as a candidate analytical tool for evaluating the performance of these complex and heterogeneous networks, preventing the use of time-consuming and onerous computer simulations. Finally, important avenues are proposed to improve the SG relevance in $6 \mathrm{G}$ networks by interacting with other powerful mathematical disciplines.

\section{REFERENCES}

[1] J. A. del Peral-Rosado, R. Raulefs, J. A. López-Salcedo, and G. SecoGranados, "Survey of cellular mobile radio localization methods: From $1 \mathrm{G}$ to 5G," IEEE Commun. Surveys Tuts, vol. 20, no. 2, pp. 1124-1148, Secondquarter 2018.

[2] M. Agiwal, A. Roy and N. Saxena, "Next generation 5G wireless networks: A comprehensive survey," IEEE Commun. Surveys Tuts, vol. 18, no. 3, pp. 1617-1655, Thirdquarter 2016.

[3] L. Zhang, Y. Liang and D. Niyato, "6G visions: Mobile ultra-broadband, super internet-of-things, and artificial intelligence," China Commun., vol. 16, no. 8, pp. 1-14, Aug. 2019

[4] P. Yang, Y. Xiao, M. Xiao and S. Li, "6G wireless communications: Vision and potential techniques," it IEEE Network, vol. 33, no. 4, pp. 70-75, July/August 2019.

[5] W. Saad, M. Bennis and M. Chen, "A Vision of 6G wireless systems: Applications, trends, technologies, and open research problems," it IEEE Network, to appear.

[6] ITU-R M.2370-0, IMT traffic estimates for the years 2020 to 2030, Jul. 2015.
[7] M. Katz, M. Matinmikko-Blue, and M. Latva-Aho, "6Genesis flagship program: Building the bridges towards 6G-enabled wireless smart society and ecosystem," in Proc. IEEE 10th Latin-American Conf. Commun. (LATINCOM), pp. 1-9, Guadalajara, 2018.

[8] 6G flagship university of Oulu, Key Drivers and Research Challenges for $6 G$ Ubiquitous Wireless Intelligence, [Online]. Available: http://jultika.oulu.fi/Record/isbn978-952-62-2354-4.

[9] H. Yanikomeroglu, "Integrated terrestrial/non-terrestrial $6 \mathrm{G}$ networks for ubiquitous 3D super-connectivity," in Proc. 21st ACM Int. Conf. Model., Anal. Simulation Wireless Mobile Syst., 2018, pp. 3-4.

[10] X. Huang, J. A. Zhang, R. P. Liu, Y. J. Guo and L. Hanzo, "AirplaneAided Integrated Networking for 6G Wireless: Will It Work?," IEEE Veh. Technol. Mag., vol. 14, no. 3, pp. 84-91, Sept. 2019.

[11] V. S. Sharma, R. Mehra, V. Kaulgud and S. Podder, "Extended reality in global software delivery- towards a common fabric of understanding and insights," in Proc. 14th ACM/IEEE Int. Conf. Global Soft. Eng. (ICGSE), pp. 80-81, Montreal, QC, Canada, 2019.

[12] G. Teniou, "3GPP achievements on VR \& ongoing developments on XR over 5G", 3GPP/VRIF/AIS 2nd Workshop on VR Ecosystems and Standards, "Immersive Media meets 5G", 15-16 April 2019, Culver City, U.S.A.

[13] Y. Mao, C. You, J. Zhang, K. Huang, and K. B. Letaief, "A survey on mobile edge computing: The communication perspective," IEEE Commun. Surveys Tuts., vol. 19, no. 4, pp. 2322-2358, Fourthquarter 2017.

[14] C. Liaskos, S. Nie, A. Tsioliaridou, A. Pitsillides, S. Ioannidis, and I. Akyildiz, "A new wireless communication paradigm through softwarecontrolled metasurfaces, IEEE Commun. Mag., vol. 56, no. 9, pp. 162169, Sep. 2018.

[15] B. Blaszczyszyn and H.P. Keeler, "Determinantal thinning of point processes with network learning applications", in Proc. IEEE Wireless Commun. Netw. Conf. (WCNC), Marrakech, Morocco, Apr. 2019.

[16] C. Saha, H. S. Dhillon, "Machine learning meets stochastic geometry: determinantal subset selection for wireless networks", in Proc. IEEE Globecom, Waikoloa, HI, Dec. 2019.

[17] M. Di Renzo, "Stochastic geometry modeling and analysis of multi-tier millimeter wave cellular networks," IEEE Trans. Wireless Commun., vol. 14, no. 9, pp. 5038-5057, Sep. 2015.

[18] J. Kokkoniemi, J. Lehtomki, and M. Juntti, "Stochastic geometry analysis for mean interference power and outage probability in $\mathrm{THz}$ networks," IEEE Trans. Wireless Commun., vol. 16, no. 5, pp. 30173028, May 2017.

[19] H. Tabassum and E. Hossain, "Coverage and rate analysis for coexisting RF/VLC downlink cellular networks," IEEE Trans. Wireless Commun., vol. 17 , no. 4, pp. 2588-2601, Apr. 2018.

[20] M. Lahmeri, M. A. Kishk, and M. -S. Alouini, "Stochastic Geometrybased analysis of Airborne Base Stations with Laser-powered UAVs," IEEE Commun. Lett., vol. 24, no. 1, pp. 173-177, Jan. 2020.

[21] Y. Deng, A. Noel, W. Guo, A. Nallanathan, and M. Elkashlan, "Analyzing large-scale multiuser molecular communication via 3-D stochastic geometry," IEEE Trans. Molecular, Biological and MultiScale Commun., vol. 3, no. 2, pp. 118-133, Jun. 2017.

[22] J. Wildman, P. H. J. Nardelli, M. Latva-aho, and S. Weber, "On the joint impact of beamwidth and orientation error on throughput in directional wireless Poisson networks," IEEE Trans. Wireless Commun., vol. 13, no. 12, pp. 7072-7085, Dec. 2014.

[23] P. D. Mankar, P. Parida, H. S. Dhillon and M. Haenggi, "Downlink analysis for the typical cell in Poisson cellular networks," IEEE Commun. Lett., to appear.

[24] M. Haenggi, "The Meta distribution of the SIR in Poisson bipolar and cellular networks," IEEE Trans. Wireless Commun., vol. 15, no. 4, pp. 2577-2589, Apr. 2016.

[25] B. B. Mandelbrot, The Fractal Geometry of Nature, Henry Holt and Company, 1983

[26] C. Zuo, L. Wu, Z.-F. Zeng, H.-L. Wei, "Stochastic fractal based multiobjective fruit fly optimisation," Int. J. Appl. Math. Comput. Sci., 2017, Vol. 27, No. 2, 417-433.

[27] O. Caramello, "The unification of mathematics via Topos theory, [Online]. Available: https://arxiv.org/abs/1006.3930. 